\title{
Machine Learning Design of Plasmonic Apertures for Optical Nanotweezers
}

\author{
Neuton Li ${ }^{1}$, Vivek Raj Shrestha ${ }^{1}$, Jasper Cadusch ${ }^{2}$, Zhe $\mathrm{Xu}^{1}$ and Kenneth B. Crozier ${ }^{1,2}$ \\ 1 - School of Physics. University of Melbourne, Victoria 3010 Australia \\ 2- Department of Electrical and Electronic Engineering, University of Melbourne, Victoria 3010 Australia \\ neuton.li@unimelb.edu.au.kcrozier@unimelb.edu.au
}

\begin{abstract}
We present a new approach to design plasmonic structures for optical trapping. Using a simulated annealing algorithm, the shape of a nanoaperture is optimized. An order of magnitude increase in trapping potential is predicted. (C) 2019 The Author(s)

OCIS codes: 350.4855 Optical tweezers or optical manipulation, 250.5403 Plasmonics
\end{abstract}

\section{Introduction}

Conventional optical tweezers offer the ability to manipulate and characterize nanoparticles in a non-invasive manner but are subject to the diffraction limit. This leads to an upper bound on the gradient force on a nanoparticle in a conventional tweezer for a given laser power, as this force is proportional to the gradient of the intensity [1]. This makes the trapping of very small particles challenging. This has motivated the use of plasmonic nanostructures as these can surpass the diffraction limit and concentrate light into deeply sub-wavelength volumes. Plasmonic nanoapertures have proven effective for this task. Geometries that have been investigated include circular apertures [2], coaxial apertures [3] and double nanoholes ( $\mathrm{DNH},[4,5])$. In general, however, these geometries have been designed by human intuition. It would be interesting to investigate whether such designs can be surpassed by those produced by machine learning. This is the topic of this abstract. In specific terms, we use a metaheuristic optimization algorithm to design a nanoaperture in gold film, with the aim of maximizing a figure of merit (FOM) that comprises the electric field enhancement at the center of the nanoaperture. We show that this produces a nanoaperture design that does not have a regular shape. Simulations indicate that our design would trap a nanoparticle with a trapping potential that is $\sim 4.5$ times deeper than that produced by a DNH with the same gap width. In addition, we allow our optimization algorithm to add a ring (i.e. annulus in which gold is removed) to the design. We show that the optimized nanoaperture with ring is predicted to achieve a trapping potential $\sim 11$ times greater than that of a DNH.

\section{Methodology and Optimization Algorithm}

Our optimization algorithm takes its initial structure to be a DNH aperture designed for operation at a wavelength of $1064 \mathrm{~nm}$. To enable a fair comparison between the original DNH and our optimized design, we maintain the geometry around the gap region, i.e. the positions of the green dots of Fig. 1a are fixed. Our optimization is based on simulated annealing (SA) and can be summarized as follows. In each iteration step, the shape of the non-fixed part of the aperture (blue dots of Fig. 1a) is randomly perturbed. The electric field distribution is simulated (COMSOL Multiphysics) and the value of the FOM is determined. The algorithm then compares the FOM of the new design to that of the previous design. If the FOM is superior to the previous FOM, then the current design is accepted. If the FOM is inferior, then there is a small but non-zero probability that it is still accepted [6]. Further details are as follows. We keep the material properties entirely consistent throughout; the algorithm only alters the nanoaperture shape. The substrate is glass and the gold (in which the aperture is formed) is $100 \mathrm{~nm}$ thick. The medium above the gold (and within the nanoaperture) is water. Illumination is from the water side, and comprises an $\boldsymbol{x}$-polarized (i.e. across the nanoaperture gap) planewave at normal incidence at $\boldsymbol{\lambda}=\mathbf{1 0 6 4} \mathbf{n m}$. Our algorithm aims to maximize an FOM that consists of the electric field enhancement (EFE) at the center of the double nanohole and halfway through the gold film.

\section{Results, Discussion and Conclusions}

The results of an iteration sequence are shown as Fig. 1b. It can be seen that over successive generations, the FOM steadily rises. After 300 iterations, the field enhancement is in excess of double that of the starting design. To further illustrate the progress of the algorithm, a few selected nanoaperture shapes are included in Fig. 1b. The nanoaperture that achieves the highest FOM is that corresponding to iteration step 274. This can be considered to be the optimal design produced by our algorithm and is studied in the remainder of this abstract. To compare it to the original DNH, we present the electric field distribution of both structures as Fig. 1c-d. These are plotted halfway through the gold film. As expected, the region with the highest EFE is in the narrow gap region at the center. It can be seen that our optimal design has an EFE that is in excess of double that of the original DNH structure. 
(a)

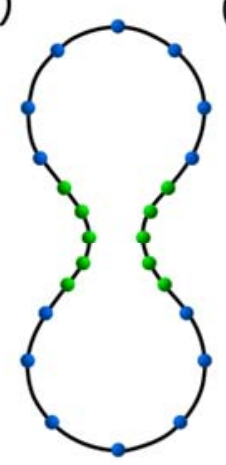

(b)

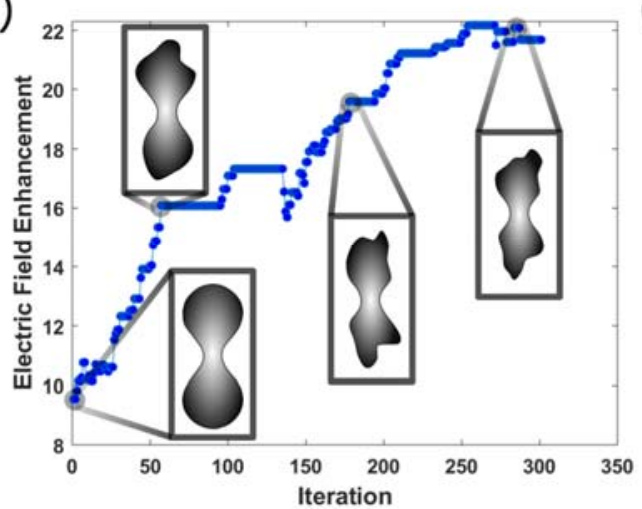

(c)

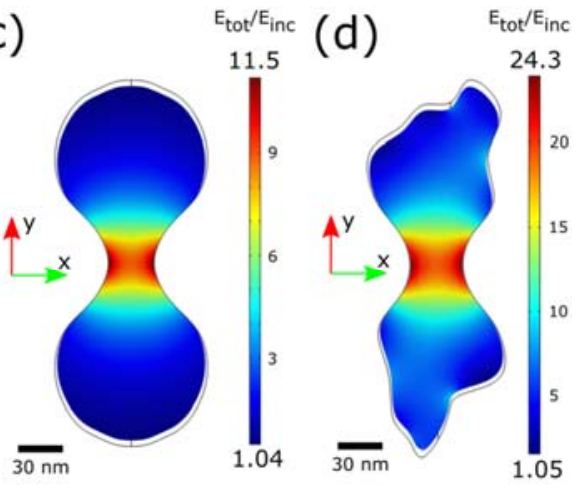

Fig. 1: (a) Control points that describe nanoaperture shape. Green points are fixed. Gold is removed from inside of shape. (b) Field enhancement vs iteration step. (c) Field enhancement of original DNH and (d) optimised structure.

We next allow the algorithm to include one or more open rings (i.e. annuli for which the gold is removed, Fig. 2a). This is motivated by the possibility that such rings might increase the coupling of incident light. Each ring is specified by its inner radius, thickness (width), gap width and gap position. A gap (i.e. where gold is not removed) is left in each ring to ensure continuity of the gold films, thereby allowing it to be effective at dissipating heat generated by optical absorption. The ring radius is constrained to be less than $600 \mathrm{~nm}$. The nanoaperture shape is not modified, i.e. the shape of Fig. 1d is used. The result of our optimization is a design containing one ring (Fig. 2b), that produces a peak EFE that is in excess of triple that of the original DNH. We next quantify the performance that might expected in an optical nanotweezer. To do so, we calculate the trapping potential for a spherical test particle (radius $5 \mathrm{~nm}$, refractive index $=1.6$ ). The trapping potential is found by integrating the gradient force in the dipole approximation [7]. As before, this is calculated halfway through the gold film. Plane wave illumination at normal incidence (xpolarized, $I_{0}=6.67 \mathrm{~mW} / \mu \mathrm{m}^{2}$ ) is employed. From Fig. 2c, it can be seen that our optimal nanoaperture (Fig. 1d) produces a potential that is deeper than that of the original DNH (Fig. 1c) by a factor of $\sim 4.5$. Furthermore, when the ring is added, the resultant design (Fig. 2b) produces a potential that is $\sim 11$ times deeper than the original DNH. In summary, we demonstrated that machine learning enables plasmonic nanotweezers to be designed that surpass those produced by traditional approaches. Our optimal design has a gap width $(30 \mathrm{~nm})$ that should be readily achievable.

(a)

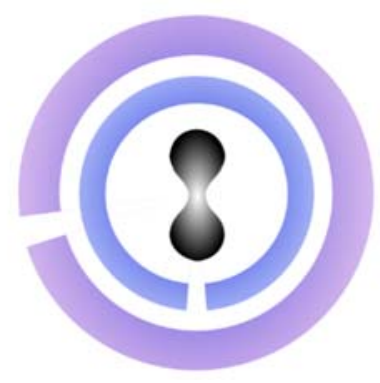

(b)

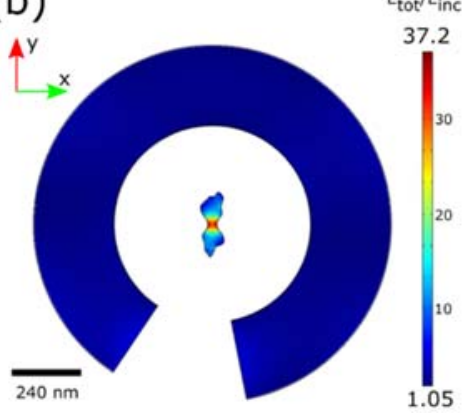

(c)

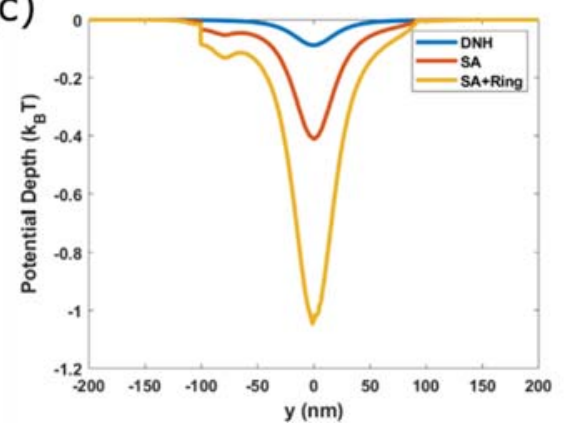

Fig. 2: (a) Schematic: nanoaperture with rings. (b) Optimized nanoaperture with ring (EFE). (c) Trapping potential vs y-position: original DNH (blue), optimized nanoaperture (red) \& optimized nanoaperture with ring (yellow).

\section{References}

[1]. P. C. Chaumet and M. Nieto-Vesperinas, “Time-averaged total force on a dipolar sphere in an electromagnetic field,” Opt. Lett. 25, 1065 (2000) [2]. E.-S. Kwak, T.-D. Onuta, D. Amarie, R. Potyrailo, B. Stein, S.C. Jacobson, W. L. Schaich, and B. Dragnea, “Optical trapping with integrated near-field apertures,” Journal of Physical Chemistry B 108, 13607 (2004)

[3] D. Yoo, K.L Gurunatha, H.-K. Choi, D. A Mohr, C. T Ertsgaard, R. Gordon, and S.-H. Oh, "Low-power optical trapping of nanoparticles and proteins with resonant coaxial nanoaperture using $10 \mathrm{~nm}$ gap,” Nano Letters 18, 3637 (2018)

[4] H. Xu, S. Jones, B.-C. Choi, and R. Gordon, "Characterization of individual magnetic nanoparticles in solution by double nanohole optical tweezers,” Nano Letters 16, 2639 (2016)

[5]. Z. Xu, W. Song, and K.B. Crozier, "Direct particle tracking observation and Brownian dynamics simulations of a single nanoparticle optically trapped by a plasmonic nanoaperture,” ACS Photonics, 5, 2850 (2018)

[6]. S. Kirkpatrick, C. D. Gelatt, and M. P. Vecchi, “Optimization by simulated annealing,” Science 220, 671 (1983)

[7] S.E.S. Spesyvtseva and K. Dholakia, “Trapping in a material world,” ACS Photonics 3, 719 (2016) 


\section{University Library}

\section{- M M N E R VA A gateway to Melbourne's research publications}

Minerva Access is the Institutional Repository of The University of Melbourne

Author/s:

Li, N;Shrestha, VR;Cadusch, J;Xu, Z;Crozier, KB

Title:

Machine learning design of plasmonic apertures for optical nanotweezers

Date:

2019-01-01

Citation:

Li, N., Shrestha, V. R., Cadusch, J., Xu, Z. \& Crozier, K. B. (2019). Machine learning design of plasmonic apertures for optical nanotweezers. Frontiers in Optics + Laser Science APS/ DLS, Part F148-LS 2019, pp.Ith3e.4-. OSA. https://doi.org/10.1364/LS.2019.LTh3E.4.

Persistent Link:

http://hdl.handle.net/11343/294881 